ARTICLE

Received 18 Jul 2013 | Accepted 7 Nov 2013 | Published 11 Dec 2013 DOl: 10.1038/ncomms3893

\title{
$5 d$ iridium oxide as a material for spin-current detection
}

Kohei Fujiwara ${ }^{1, \dagger}$, Yasuhiro Fukuma ${ }^{1, \dagger}$, Jobu Matsuno ${ }^{1, \dagger}$, Hiroshi Idzuchi ${ }^{2}$, Yasuhiro Niimi ${ }^{2}$, YoshiChika Otani ${ }^{1,2, \dagger} \&$ Hidenori Takagi,3

Devices based on pure spin currents have been attracting increasing attention as key ingredients for low-dissipation electronics. To integrate such spintronics devices into chargebased technologies, electric detection of spin currents is essential. The inverse spin Hall effect converts a spin current into an electric voltage through spin-orbit coupling. Noble metals such as $\mathrm{Pt}$ and $\mathrm{Pd}$, and also Cu-based alloys, have been regarded as potential materials for a spincurrent injector, owing to the large direct spin Hall effect. Their spin Hall resistivity $\rho_{\mathrm{SH}}$, representing the performance as a detector, is not large enough, however, due mainly because of their low charge resistivity. Here we report that a binary $5 d$ transition metal oxide, iridium oxide, overcomes the limitations encountered in noble metals and Cu-based alloys and shows a very large $\rho_{\mathrm{SH}} \sim 38 \mu \Omega \mathrm{cm}$ at room temperature.

\footnotetext{
${ }^{1}$ RIKEN Advanced Science Institute, Wako, Saitama 351-0198, Japan. ${ }^{2}$ Institute for Solid State Physics, University of Tokyo, Kashiwa, Chiba 277-8581, Japan. ${ }^{3}$ Department of Physics, University of Tokyo, Hongo, Tokyo 113-0033, Japan. †Present addresses: The Institute of Scientific and Industrial Research, Osaka University, Ibaraki, Osaka 567-0047, Japan. (K.F.); Frontier Research Academy for Young Researchers, Kyushu Institute of Technology, lizuka, Fukuoka 820-8502, Japan. (Y.F.); RIKEN Center for Emergent Matter Science (CEMS), Wako, Saitama 351-0198, Japan. (J.M., Y.O.). Correspondence and requests for materials should be addressed to K.F. (email: kfujiwara@sanken.osaka-u.ac.jp).
} 
S pin-orbit coupling (SOC) is a relativistic effect and relates the spin moment of an electron to its orbital momentum via a momentum-dependent effective magnetic field. In the presence of SOC, a charge current without any spin polarization can be converted into a pure spin current (the flow of spin angular momentum) and vice versa, known as the direct spin Hall effect and the inverse spin Hall effect (ISHE) ${ }^{1-18}$. The presence of these phenomena in non-magnetic semiconductors had already been recognized in 1970s (refs 19-22) but recent theoretical ${ }^{1,2}$ and experimental investigations ${ }^{3,4}$ have given a boost to this field. Spin current-based electronics with low-energy consumption has since been discussed, where spin-current injection and detection, using charge/spin conversion by direct spin Hall effect and ISHE, played a key role. A variety of materials have subsequently been explored to realize efficient charge/spin conversion ${ }^{5-18}$. The playground for the exploration was first limited to semiconductors but then extended to metals, where we can take advantage of the less pronounced effect of interfacial barriers. Heavy transition metals such as $\mathrm{Pt}^{6-10,14}, \mathrm{Au}^{9,12,13}$, and $\mathrm{Pd}^{8,9,11}$ and also $\mathrm{Cu}$-based alloys ${ }^{17,18}$ were found to exhibit a particularly large spin Hall angle $\alpha_{\mathrm{SH}}$ (the maximum yield of the charge/spin conversion), $0.01-0.1$ at room temperature, owing to their pronounced SOC effects. These effects give these metals an advantage for application as a spin injector. In addition to the large $\alpha_{\mathrm{SH}}$, their low electrical resistivity $\left(\rho_{\mathrm{C}}\right)$, typically $\rho_{\mathrm{C}}=10^{-7}-10^{-5} \Omega \mathrm{cm}$, allows large charge currents to be passed and hence to inject a large spin current without serious Joule heating.

Spin currents can be detected by using the ISHE. In contrast to the case for spin injectors, the low $\rho_{\mathrm{C}}$ of the heavy metals with a large $\alpha_{\mathrm{SH}}$ is a disadvantages when it comes to spin detection. In the ISHE, the electric voltage $\Delta V_{\text {ISHE }}$ generated by a spin current $I_{\mathrm{S}}$ is in proportion to $\rho_{\mathrm{C}}$ (refs 23,24$)$ :

$$
\Delta V_{\text {ISHE }} \propto \alpha_{\mathrm{SH}} \rho_{\mathrm{C}} I_{\mathrm{S}} \approx \rho_{\mathrm{SH}} I_{\mathrm{S}} .
$$

Spin Hall resistivity given by $\rho_{\mathrm{SH}} \approx \alpha_{\mathrm{SH}} \rho_{\mathrm{C}}$ determines the efficiency of spin-current detection. This means that a sensitive detection of spin current could be achieved in materials with both a large $\alpha_{\mathrm{SH}}$ and a high $\rho_{\mathrm{C}}$. In typical heavy metals, their low $\rho_{\mathrm{C}}$ imposes strong constraints in tuning the value of $\rho_{\mathrm{SH}}$ as it remains as low as $<1 \mu \Omega \mathrm{cm}$. One of the obvious approaches to improve the performance of spin detectors could be to increase the $\rho_{\mathrm{C}}$ by alloying. Alloying can in fact increase not only the $\rho_{\mathrm{C}}$ but also the $\alpha_{\mathrm{SH}}$ through an extrinsic spin Hall effect ${ }^{25-27}$. At room temperature where the inelastic scattering of charge carriers is dominant, however, it should be hard to anticipate a drastic increase of $\rho_{\mathrm{C}}$ by alloying, for example, more than an order of magnitude increase as compared with the pristine material. Therefore, the improvement of $\rho_{\mathrm{SH}}$ would not be achieved only by alloying.

In this work, we propose $5 d$ transition metal oxides (TMOs) as alternative metals to realize both large $\alpha_{\mathrm{SH}}$ and large $\rho_{\mathrm{C}}$, which should enhance $\rho_{\mathrm{SH}}$. The uniqueness of $5 d$ TMOs is characterized by the extremely strong SOC $\sim 0.5-1 \mathrm{eV}$ originating from the predominant $5 d$ character of the conduction band. The SOC in $5 d$ TMOs is in fact as strong as to reconstruct the electronic structures drastically, as first discussed in the case of $J_{\text {eff }}=1 / 2$ Mott state $\mathrm{Sr}_{2} \mathrm{IrO}_{4}$ (ref. 28). The analogous predominant SOC effect has been commonly observed in a broad range of Ir oxides including $\mathrm{Ba}_{2} \mathrm{IrO}_{4}$ (ref. 29), $\mathrm{CaIrO}_{3}$ (ref. 30) and $\mathrm{Ir}_{2} \mathrm{O}_{4}$ (ref. 31). In those Ir oxides, in addition to the strong SOC, the localized character of $d$ orbitals gives rise to a moderately high charge resistivity $\rho_{\mathrm{C}}$ even in the metallic state. Typical $\rho_{\mathrm{C}}$ values of conductive $5 d$ Ir TMOs such as $\mathrm{IrO}_{2}$ and $\mathrm{SrIrO}_{3}$ at room temperature are of the order of $10^{-4}-10^{-3} \Omega \mathrm{cm}$, at least one order of magnitude higher than those of normal $s$ metals. As the variety of materials continues to grow rapidly, $5 d$ TMOs offer a unique opportunity to explore the giant $\rho_{\mathrm{SH}}$ for efficient spin detection. We selected a simple binary oxide, rutile $\mathrm{IrO}_{2}$ (refs 32-34), as the basis for our exploration. $\mathrm{IrO}_{2}$ with $\mathrm{Ir}^{4+}$ shares the same $5 d^{5}$ configuration with many other Ir oxides, where the strong SOC dominates the electronic states and the resultant electronic properties ${ }^{34}$. This binary oxide has been long used as an electrode in various device applications, ranging from non-volatile ferroelectric memories to electrochemical devices. The excellent electrode properties are due to the formation of a clean, well-defined interface with other materials owing to its high chemical and thermal stability, and superior barrier properties for oxygen diffusion. In such a clean interface, it may be possible to inject a spin current through a diffusion process; $\mathrm{IrO}_{2}$ is therefore an ideal platform for testing the ISHE of $5 d$ TMOs. Here, we describe successful spin-current injection into $\mathrm{IrO}_{2}$ using a lateral spin-valve (LSV) device geometry and demonstrate the ISHE with a remarkably high $\rho_{\mathrm{SH}}$ as a metal not only in the polycrystalline state but also in the practically important amorphous state.

\section{Results}

Device structure. We fabricated cross-junction-type devices consisting of an $\mathrm{IrO}_{2}$ wire and a permalloy $\left(\mathrm{Ni}_{80} \mathrm{Fe}_{20}, \mathrm{Py}\right) / \mathrm{Ag} / \mathrm{Py}$ $\mathrm{LSV}^{35}$, shown in Fig. 1a,b (see also Methods). Spin currents are passed in the Ag strip by a non-local charge current injection $\left(I_{\mathrm{C}}\right)$ from the ferromagnetic Py electrode and are perpendicularly injected in part into the $\mathrm{IrO}_{2}$ wire through the diffusion (Fig. 1a). This effect, called spin absorption, enables us to conduct a quantitative analysis of the ISHE as have been demonstrated for various materials $7,8,17,18$. We examined the potential of polycrystalline and amorphous $\mathrm{IrO}_{2}$ wires as the spin detector element. Their resistivities were $2.0 \times 10^{-4} \Omega \mathrm{cm}$ and $5.7 \times 10^{-4} \Omega \mathrm{cm}$ at $300 \mathrm{~K}$, respectively, which are $1-2$ orders of magnitude higher than those of metals that have been studied to date as a spin Hall material.
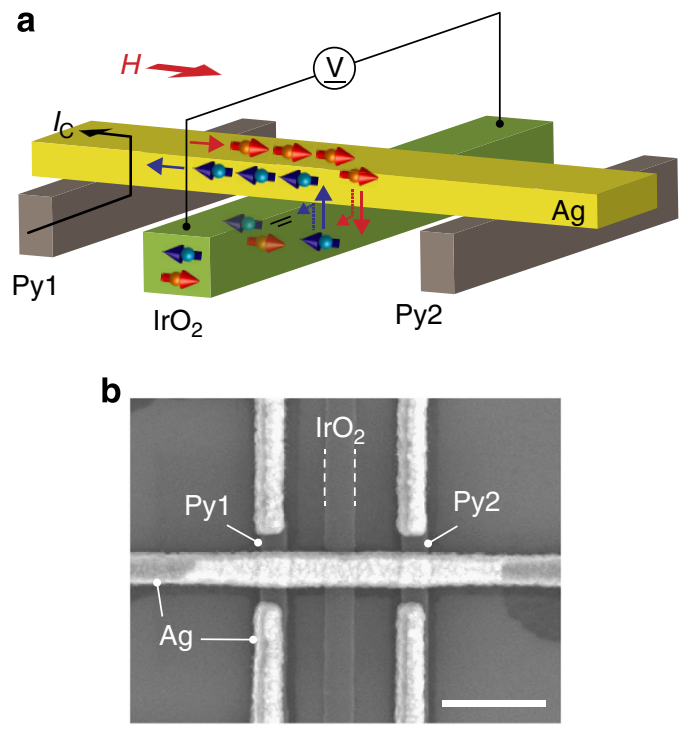

Figure 1 | Inverse spin Hall effect measurements using a spin absorption effect in a lateral spin-valve geometry. (a) The structure of device used for ISHE measurements is shown schematically. A LSV with two Py electrodes (Py1: spin source, Py2: spin detector) bridged by an Ag spin transport layer was formed on an $\mathrm{IrO}_{2}$ wire. Spin-polarized charge current, $I_{C}$, was injected along the arrow to accumulate pure spin currents in the $\mathrm{Ag}$. The diffusion of spin current into the $\mathrm{IrO}_{2}$ wire, namely, the spin absorption, gives rise to the ISHE in $\mathrm{IrO}_{2}$. (b) Scanning electron microscopy image of a typical device. Scale bar, $500 \mathrm{~nm}$. The dotted lines indicate the side edges of the $\mathrm{IrO}_{2}$ wire for clarity. 
To check that the Ag layer is not oxidized by directly contacting with $\mathrm{IrO}_{2}$, we first characterized the $\mathrm{Ag} / \mathrm{IrO}_{2}$ interface by means of interface resistance measurements ${ }^{36}$. The currentvoltage characteristics of the $\mathrm{Ag} / \mathrm{IrO}_{2}$ interface showed an ohmic behaviour, typical of metal/metal interface. The slope of the data yielded a resistance-area product (RA) of $2.3 \mathrm{f} \Omega \mathrm{m}^{2}$ at $300 \mathrm{~K}$, which is as low as that of the metallic, transparent $\mathrm{Ag} / \mathrm{Py}$ interface $\left(\mathrm{RA}=1.9 \mathrm{f} \Omega \mathrm{m}^{2}\right.$ at $\left.300 \mathrm{~K}\right)$. An estimate of the thickness of the possibly oxidized $\mathrm{Ag}$ layer, using the resistivities of $\mathrm{Ag}_{2} \mathrm{O}$ and $\mathrm{AgO}$ in the literature ${ }^{37}$, never exceeded $0.1 \mathrm{~nm}$. These observations imply that the $\mathrm{Ag} / \mathrm{IrO}_{2}$ interface in the present devices is very sharp as in metal/metal interfaces.

Spin-current injection into $\mathrm{IrO}_{2}$. The spin absorption by $\mathrm{IrO}_{2}$ across the interface was then confirmed by non-local spin-valve (NLSV) experiments $7,8,17,18,35$. The schematic diagram of the measurement is shown in Fig. 2a. The pure spin currents accumulated in the Ag strip by injecting spin-polarized charge currents $I_{\mathrm{C}}$ from $\mathrm{Py} 1$ to $\mathrm{Ag}$ is detected as a voltage $V_{\mathrm{S}}$ $\left(=V_{+}-V_{-}\right)$between Py2 $\left(V_{+}\right)$and $\mathrm{Ag}\left(V_{-}\right)$(upper panel). The spin accumulation signal $\Delta R_{\mathrm{S}}$ is given by the difference in $V_{\mathrm{S}}$ / $I_{\mathrm{C}}\left(R_{\mathrm{S}}\right)$ between parallel (high $R_{\mathrm{S}}$ ) and antiparallel (low $R_{\mathrm{S}}$ ) magnetizations of the two Py wires. If an $\mathrm{IrO}_{2}$ middle wire is in contact with the Ag, the accumulated spin diffuses in part into it (lower panel), resulting in the reduction in $\Delta R_{\mathrm{S}}$ (ref. 7). Figure $2 \mathrm{~b}$ and $\mathrm{c}$ show field dependences of $R_{\mathrm{S}}$ at 300 and $10 \mathrm{~K}$, respectively. The decrease of $\Delta R_{\mathrm{S}}$ by the insertion of the $\mathrm{IrO}_{2}$ middle wire indicates the spin absorption effect. This is more clearly demonstrated in the temperature $(T)$ dependence of $\Delta R_{\mathrm{S}}$ with $\left(\Delta R_{\mathrm{S}}^{\text {with }}\right)$ and without $\left(\Delta R_{\mathrm{S}}^{\text {without }}\right)$ the middle wire, as displayed in Fig. 2d. At all the temperatures, $\Delta R_{\mathrm{S}}^{\text {with }}$ is systematically smaller than $\Delta R_{\mathrm{S}}^{\text {without }}$, while both are enhanced at low temperatures owing to the increase of the spin-diffusion length of $\mathrm{Ag}\left(\lambda_{\mathrm{Ag}}\right)^{35}$. By analysing the data based on the threedimensional spin-diffusion model ${ }^{18}$ (see Supplementary Methods and Supplementary Fig. S1), we estimated a spin-diffusion length in polycrystalline $\mathrm{IrO}_{2}, \lambda_{\mathrm{IrO}_{2}}=3.8 \mathrm{~nm}$ at $300 \mathrm{~K}$ and $8.4 \mathrm{~nm}$ at $10 \mathrm{~K}$, which are comparable to those of Pt $(3-10 \mathrm{~nm}$ at $300 \mathrm{~K}$ (refs 6-10,14)) and CuIr (5-20 nm at $10 \mathrm{~K}$ (ref. 17)) where relatively large spin Hall angles were observed. This indicates that spin scattering of $\mathrm{IrO}_{2}$ is indeed very strong as expected from the $5 d$ conduction band ${ }^{34}$.

ISHE and $\rho_{\mathrm{SH}}$ of $\mathrm{IrO}_{2}$. By injecting spin currents through the spin absorption effect, we successfully observed ISHE in $\mathrm{IrO}_{2}$. In Fig. 3a,b, we plot the room-temperature ISHE signal $R_{\mathrm{ISHE}}$ ( $\left.\equiv \Delta V_{\text {ISHE }} / I_{\mathrm{C}}\right)$ for polycrystalline and amorphous samples as a function of the magnetic field (The temperature dependence of the ISHE is shown in Supplementary Fig. S2 and is explained in Supplementary Note 1). Here the magnetic field was applied along the hard axis of the Py spin source (see Fig. 1a), and $\Delta V_{\text {ISHE }}$ was induced along the long-axis direction of the $\mathrm{IrO}_{2}$ wire. Reflecting the magnetization process in hard axis of Py, $R_{\mathrm{ISHE}}$ linearly increased up to $\sim 1,500$ Oe and then saturated. The antisymmetric response to the applied magnetic field excludes any spurious effects such as anisotropic magnetoresistance of $\mathrm{Py}$; the ISHE of $\mathrm{IrO}_{2}$ is responsible for the resistance change $\left(2 \Delta R_{\text {ISHE }}\right)$ between positive and negative magnetic fields. The in-plane angular dependence of magnetic field effect on $R_{\mathrm{ISHE}}$ was confirmed fully consistent with the ISHE origin. Figure $3 \mathrm{c}$ shows the variation in $R_{\mathrm{ISHE}}$ under a magnetic field direction rotated by $\phi$ with respect to the ordinary set-up (Fig. $3 \mathrm{~d}$, inset). The sign change of $R_{\mathrm{ISHE}}$ occurs between $\phi=0$ and $\pi$ by magnetization switching of Py and concomitant polarization reversal of the spin a
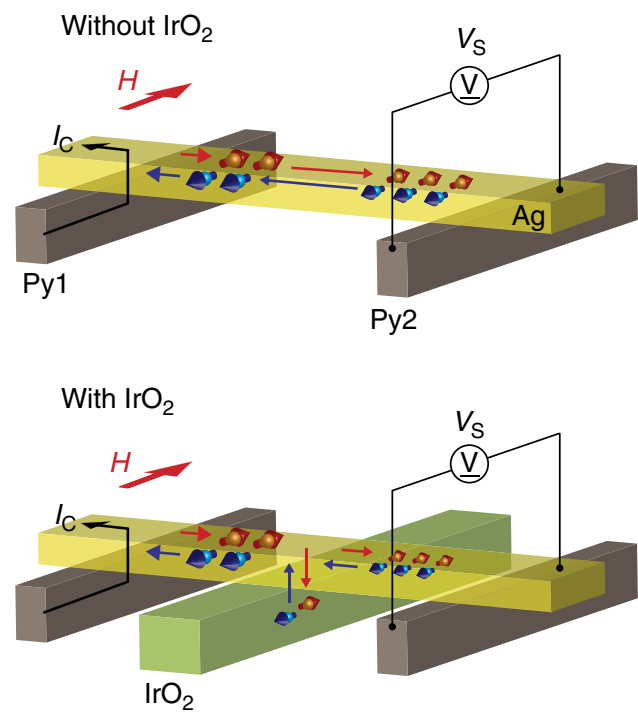

b
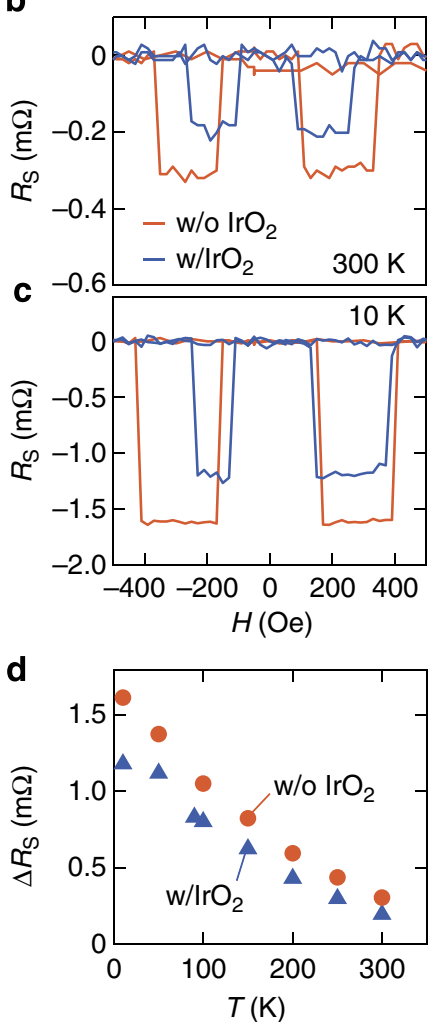

Figure 2 | Non-local spin-valve measurements. (a) The set-up of measurement is schematically depicted. The magnetic field $H$ is applied along the easy axis of the Py electrodes. (b,c) NLSV signals measured at $300 \mathrm{~K}$ and $10 \mathrm{~K}$ for Py/Ag/Py LSVs without and with the polycrystalline IrO $\mathrm{I}_{2}$ middle wire. (d) Temperature dependences of the NLSV signals. 

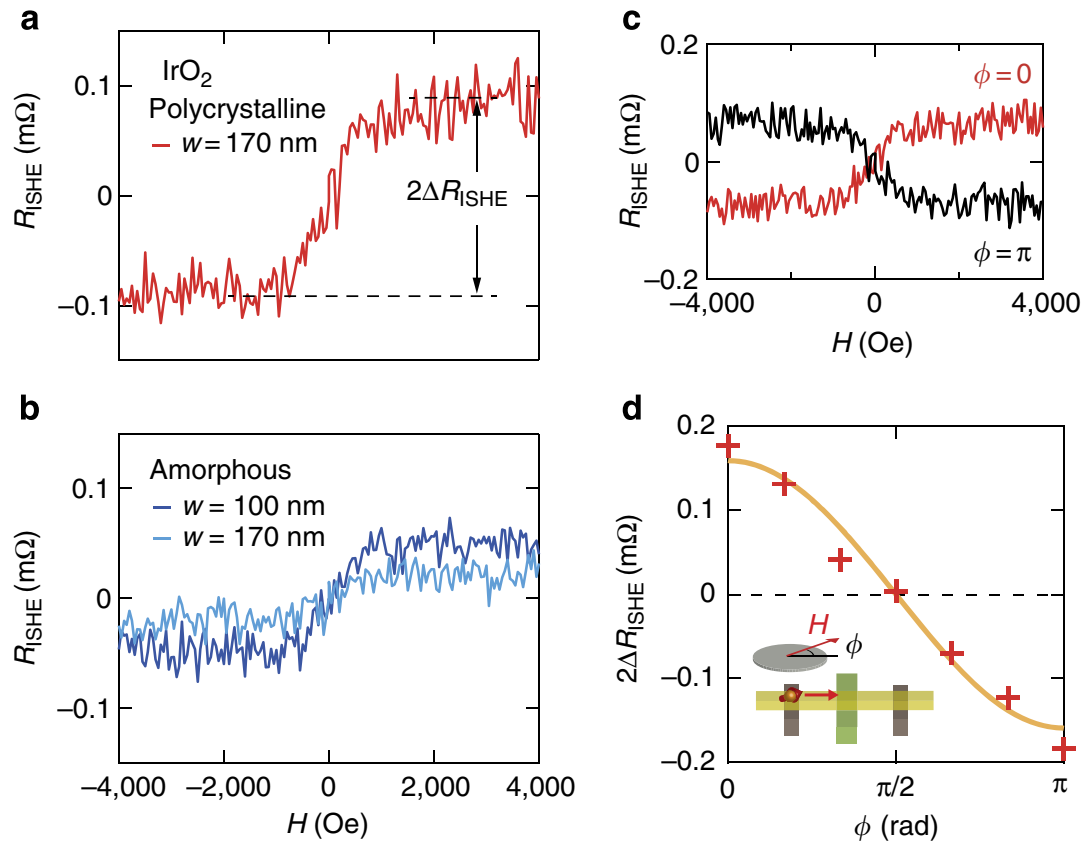

Figure 3 | Inverse spin Hall effect of $\mathrm{IrO}_{\mathbf{2}}$ at $\mathbf{3 0 0} \mathbf{K}$. (a) The ISHE signal $R_{\mathrm{ISHE}}\left(\equiv \Delta V_{\mathrm{ISHE}} / \mathrm{I}_{\mathrm{C}}\right)$ for a polycrystalline $\mathrm{IrO}_{2}$ wire with a width $w$ of $170 \mathrm{~nm}$ was measured as a function of the applied magnetic field $\mathrm{H}$. (b) The results for amorphous $\mathrm{rO}_{2}$ samples. Two devices with different $w$ values (100 and $170 \mathrm{~nm}$ ) were measured. The $100 \mathrm{~nm}$-width device showed larger $R_{\text {ISHE }}$ because of the increase of spin-current density. $R_{\text {ISHE }}$ of the two amorphous devices are smaller than that of the polycrystalline sample shown in $\mathbf{a}$. (c,d) Angular dependence of $R_{\mathrm{ISHE}}$ and the resistance change $2 \Delta R_{\mathrm{ISHE}}$ by the ISHE. The in-plane azimuthal angle $\phi$ with respect to the long axis of $\mathrm{Ag}$ is rotated to change the polarization direction of the spin current (inset in $\mathbf{d}$ ). The solid line in $\mathbf{d}$ is a $\cos \phi$ curve fitted to the results.

current. As shown in Fig. $3 \mathrm{~d}$, the resistance change $2 \Delta R_{\mathrm{ISHE}}$ vanishes at $\phi=\pi / 2$ and exhibits a $\cos \phi$ relation. This characteristic angular dependence is the hallmark of $\operatorname{ISHE}^{7,12}$, evidencing spin-dependent electron scattering in $\mathrm{IrO}_{2}$.

Because of the large difference in $\rho_{\mathrm{C}}$ between $\mathrm{Ag}$ and $\mathrm{IrO}_{2}$, the charge current induced in the $\mathrm{IrO}_{2}$ wire by the ISHE is partially shunted by the adjacent Ag layer. To take into account such geometric effects and to precisely determine $\rho_{\mathrm{SH}}$, we adopted the three-dimensional spin-diffusion model (see also Supplementary Methods). Using the ISHE signal $2 \Delta R_{\mathrm{ISHE}}$ and data obtained from the NLSV measurements, $\alpha_{\mathrm{SH}}$ and $\rho_{\mathrm{SH}}$ are, respectively, calculated to be 0.040 and $8.0 \mu \Omega \mathrm{cm}$ for polycrystalline $\mathrm{IrO}_{2}$, and 0.065 and $37.5 \mu \Omega \mathrm{cm}$ for amorphous $\mathrm{IrO}_{2}$ at $300 \mathrm{~K}$.

Figure 4 summarizes spin Hall resistivity $\rho_{\mathrm{SH}}$ and electrical resistivity $\rho_{\mathrm{C}}$ for a variety of metals with a large spin Hall angle $\alpha_{\mathrm{SH}}$ reported so far, together with that for $\mathrm{IrO}_{2}$. It mimics the point of this work in that $\rho_{\mathrm{SH}}$ of $\mathrm{IrO}_{2}$ is distinctly large compared with typical heavy metals and their alloys of which $\rho_{\mathrm{SH}}$ is $\sim 0.16-1.2 \mu \Omega \mathrm{cm}$ (refs $6-14,17,18$ ), and is now comparable to the large $\rho_{\mathrm{SH}}$ that was recently discovered in ultrathin films of $\beta$-phase (A15 crystal structure) W (ref. 15) and Ta (ref. 16).

\section{Discussion}

To generate a large electric voltage using the ISHE of $\mathrm{IrO}_{2}$ from existing metal-based spin-current devices, it is important to reduce the shunting effect at the $\mathrm{Ag} / \mathrm{IrO}_{2}$ interface due to their large difference in $\rho_{\mathrm{C}}$. One of the promising approaches is to use $\mathrm{Al}$ (ref. 38) and $\mathrm{Mg}$ (ref. 39) as the non-magnetic spin transport layer. Their $\rho_{\mathrm{C}}$ values are one order of magnitude higher than that of $\mathrm{Ag}$ we used, of the order of $10 \mu \Omega \mathrm{cm}$ at room temperature, while a comparably long spin-diffusion length is obtained. Another interesting route is a spin transport layer based on a conductive oxide composed of light elements with small
SOC. For example, in $\mathrm{ZnO}$ (ref. 40), doping with $\mathrm{Al}$ yields $\rho_{\mathrm{C}}$ of the order of $100 \mu \Omega \mathrm{cm}$ (ref. 41), which can match with that of $\mathrm{IrO}_{2}$. The high chemical stability of $\mathrm{IrO}_{2}$ could be advantageous in fabricating such oxide-based junctions.

The discovery of the ISHE in amorphous $\mathrm{IrO}_{2}$, which can be grown at room temperature, is a significant step towards spintronics devices based on such oxide materials. The comparably large $\alpha_{\mathrm{SH}}$ in the amorphous form may point to that spin scattering in this compound occurs at the length scale of as short as a unit cell, potentially enabling the spin detection at the atomic layer thickness. Although the physics of spin scattering in amorphous states has not been understood yet, the availability of the amorphous form should make it easier to study SOC-induced transport phenomena in $5 d$ TMOs not used before in spintronics.

In conclusion, $5 d$ TMOs are the most promising materials for spin detection in spintronics. We demonstrated that the combination of strong SOC and a moderately high $\rho_{\mathrm{C}}$ is a key to improve the performance of spin detection. These achievements in the simple binary oxide manifest the potential of $5 d$ TMOs as a new class of spintronic materials. Strong SOC in this class of materials produces a rich variety of intriguing physical properties, including correlated topological insulator ${ }^{42}$, Weyl semi-metal ${ }^{43}$ and Kitaev spin liquid ${ }^{44}$. Our discovery of the large spin Hall resistivity may make $5 d$ TMOs even more fascinating.

\section{Methods}

Device fabrication. The Py/Ag/Py LSV with an $\mathrm{IrO}_{2}$ middle wire was fabricated on a $\mathrm{SiO}_{2} / \mathrm{Si}$ substrate. $\mathrm{Au} / \mathrm{Ti}$ leads for electrical measurements were prepared on the substrate by a photolithography and an e-beam deposition. $\mathrm{IrO}_{2}$ film was grown by a reactive sputtering with a $99.9 \%$ pure Ir target. A wire structure of $\mathrm{IrO}_{2}$ was formed using a posi-type resist patterned by an e-beam lithography. The typical wire width and thickness were 170 and $15 \mathrm{~nm}$, respectively. During the deposition, the substrate was not heated, and the pressure was fixed at $0.7 \mathrm{~Pa}$ of $\mathrm{Ar} / \mathrm{O}_{2}$ (90:10) mixture gas. X-ray diffraction analysis indicated that the as-deposited $\mathrm{IrO}_{2}$ film was amorphous. The amorphous film turned into 


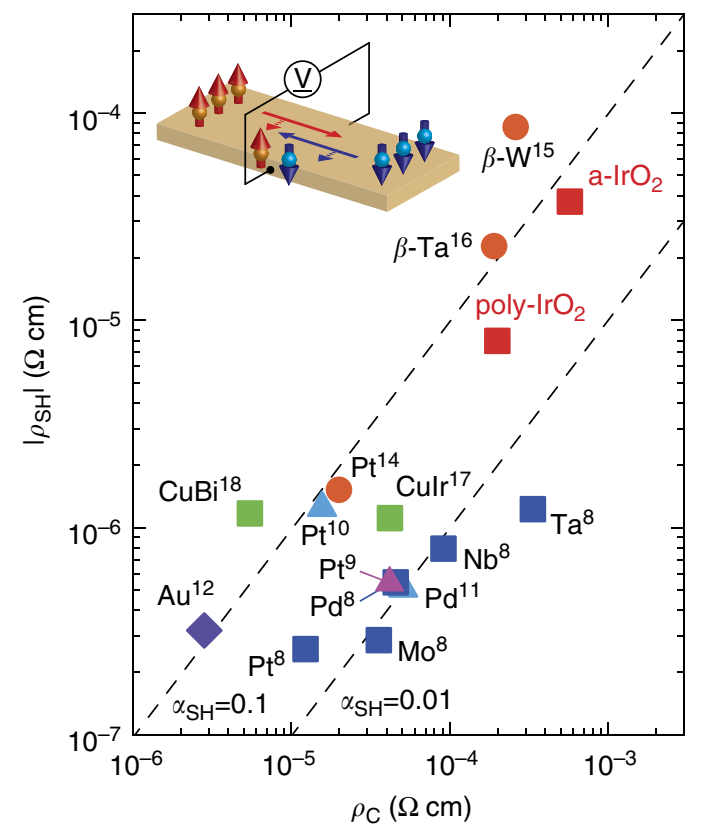

Figure 4 | Spin Hall resistivity of metals. Experimentally measured values of spin Hall resistivity $\rho_{\mathrm{SH}}$ for various metals are plotted as a function of electrical resistivity $\rho_{\mathrm{C}}$. Data include Pt (refs 9,10) and Pd (ref. 11) evaluated at room temperature by spin pumping, Pt (ref. 14) and atomic layer thin films of $\beta-W$ (ref. 15) and $\beta$-Ta (ref. 16) at room temperature by spintransfer-torque ferromagnetic resonance, $\mathrm{Au}$ at $295 \mathrm{~K}$ using a planar Hall device ${ }^{12}, \mathrm{Ta}, \mathrm{Nb}, \mathrm{Pd}, \mathrm{Mo}$, Pt (ref. 8), 12\% Ir-doped Cu (ref. 17), 0.5\% Bidoped $\mathrm{Cu}$ (ref. 18) at $10 \mathrm{~K}$, and polycrystalline and amorphous $\mathrm{IrO}_{2}$ at $300 \mathrm{~K}$ (this work) by spin absorption.

polycrystalline after a post-annealing at $400{ }^{\circ} \mathrm{C}$ in air. The LSV with a Py-Py separation of $700 \mathrm{~nm}$ (centre-to-centre) was then formed onto the $\mathrm{IrO}_{2}$ wire by the shadow evaporation method ${ }^{35,36}$. To prevent the surface oxidation of Ag, a 2-nmthick $\mathrm{MgO}$ protection layer was e-beam deposited on the surface without breaking vacuum. The width and thickness of wire were 170 and $100 \mathrm{~nm}$ for $\mathrm{Ag}$, and 170 and $20 \mathrm{~nm}$ for Py, respectively. Their $\rho_{\mathrm{C}}$ and spin-diffusion length were $3.0 \mu \Omega \mathrm{cm}$ and $330 \mathrm{~nm}$ for $\mathrm{Ag}$ and $47 \mu \Omega \mathrm{cm}$ and $5 \mathrm{~nm}$ for Py, respectively, at $300 \mathrm{~K}$. One of the two Py electrodes was made longer than the other (not shown in Fig. 1b) to make the contrast in the fields for magnetization reversal.

Electrical measurements. All electrical measurements were performed by a standard lock-in technique in a He flow cryostat. An alternating current $I_{\mathrm{C}}$ of $200 \mu \mathrm{A}$ with a frequency of $79 \mathrm{~Hz}$ was used for NLSV measurements. In ISHE measurements, $I_{\mathrm{C}}$ of $400 \mu \mathrm{A}$ was applied. We confirmed the linearity between the output voltage $\Delta V_{\text {ISHE }}$ and $I_{\mathrm{C}}$ in the range of $100-400 \mu \mathrm{A}$.

\section{References}

1. Hirsch, J. Spin Hall effect. Phys. Rev. Lett. 83, 1834-1837 (1999).

2. Zhang, S. Spin Hall effect in the presence of spin diffusion. Phys. Rev. Lett. 85, 393-396 (2000).

3. Kato, Y. K., Myers, R. C., Gossard, A. C. \& Awschalom, D. D. Observation of the spin Hall effect in semiconductors. Science 306, 1910-1913 (2004).

4. Wunderlich, J., Kaestner, B., Sinova, J. \& Jungwirth, T. Experimental observation of the spin-Hall effect in a two-dimensional spin-orbit coupled semiconductor system. Phys. Rev. Lett. 94, 047204 (2005).

5. Valenzuela, S. O. \& Tinkham, M. Direct electronic measurement of the spin Hall effect. Nature 442, 176-179 (2006).

6. Saitoh, E., Ueda, M., Miyajima, H. \& Tatara, G. Conversion of spin current into charge current at room temperature: inverse spin-Hall effect. Appl. Phys. Lett. 88, 182509 (2006).

7. Kimura, T., Otani, Y., Sato, T., Takahashi, S. \& Maekawa, S. Room-temperature reversible spin Hall effect. Phys. Rev. Lett. 98, 156601 (2007).

8. Morota, M. et al. Indication of intrinsic spin Hall effect in $4 \mathrm{~d}$ and $5 \mathrm{~d}$ transition metals. Phys. Rev. B 83, 174405 (2011).

9. Mosendz, O. et al. Detection and quantification of inverse spin Hall effect from spin pumping in permalloy/normal metal bilayers. Phys. Rev. B 82, 214403 (2010).
10. Ando, K. et al. Electric manipulation of spin relaxation using the spin Hall effect. Phys. Rev. Lett. 101, 036601 (2008).

11. Ando, K. \& Saitoh, E. Inverse spin-Hall effect in palladium at room temperature. J. Appl. Phys. 108, 113925 (2010).

12. Seki, T. et al. Giant spin Hall effect in perpendicularly spin-polarized $\mathrm{FePt} / \mathrm{Au}$ devices. Nat. Mater. 7, 125-129 (2008).

13. Mihajlović, G., Pearson, J., Garcia, M., Bader, S. \& Hoffmann, A. Negative nonlocal resistance in mesoscopic gold Hall bars: absence of the giant spin Hall effect. Phys. Rev. Lett. 103, 166601 (2009).

14. Liu, L., Moriyama, T., Ralph, D. \& Buhrman, R. Spin-torque ferromagnetic resonance induced by the spin Hall effect. Phys. Rev. Lett. 106, 036601 (2011).

15. Pai, C.-F. et al. Spin transfer torque devices utilizing the giant spin Hall effect of tungsten. Appl. Phys. Lett. 101, 122404 (2012).

16. Liu, L. et al. Spin-torque switching with the giant spin Hall effect of tantalum. Science 336, 555-558 (2012)

17. Niimi, Y. et al. Extrinsic spin Hall effect induced by iridium impurities in copper. Phys. Rev. Lett. 106, 126601 (2011).

18. Niimi, Y. et al. Giant spin Hall effect induced by skew scattering from bismuth impurities inside thin film CuBi alloys. Phys. Rev. Lett. 109, 156602 (2012).

19. Chazalviel, J.N. \& Solomon, I. Experimental evidence of the anomalous Hall effect in a nonmagnetic semiconductor. Phys. Rev. Lett. 29, 1676-1679 (1972).

20. Chazalviel, J.N. Spin-dependent Hall effect in semiconductors. Phys. Rev. B 11, 3918-3934 (1975).

21. D'yakonov, M. I. \& Perel', V. I. Possibilty of orienting electron spins with current. Sov. Phys. JETP Lett. 13, 467-469 (1971)

22. Bakun, A. A., Zakharchenya, B. P., Rogachev, A. A., Tkachuk, M. N. \& Flěsher, V. G. Observation of a surface photocurrent caused by optical orientation of electons in a semiconductor. Sov. Phys. JETP Lett. 40, 1293-1295 (1984).

23. Ando, K. \& Saitoh, E. Observation of the inverse spin Hall effect in silicon. Nat. Comm. 3, 629 (2012)

24. Vila, L., Kimura, T. \& Otani, Y. Evolution of the spin Hall effect in Pt nanowires: size and temperature effects. Phys. Rev. Lett. 99, 226604 (2007).

25. Gu, B. et al. Surface-assisted spin Hall effect in Au films with Pt impurities. Phys. Rev. Lett. 105, 216401 (2010).

26. Fert, A. \& Levy, P. Spin Hall effect induced by resonant scattering on impurities in metals. Phys. Rev. Lett. 106, 157208 (2011).

27. Gradhand, M. et al. Perfect alloys for spin Hall current-induced magnetization switching. SPIN 2, 1250010 (2012).

28. Kim, B. J. et al. Phase-sensitive observation of a spin-orbital Mott state in $\mathrm{Sr}_{2} \mathrm{IrO}_{4}$. Science 323, 1329-1332 (2009).

29. Okabe, $\mathrm{H}$. et al. $\mathrm{Ba}_{2} \mathrm{IrO}_{4}$ : a spin-orbit Mott insulating quasi-two-dimensional antiferromagnet. Phys. Rev. B 83, 155118 (2011).

30. Ohgushi, K. et al. Resonant X-ray diffraction study of strongly spin-orbitcoupled Mott insulator $\mathrm{CaIrO}_{3}$. Phys. Rev. Lett. 110, 217212 (2013).

31. Kuriyama, H. et al. Epitaxially stabilized iridium spinel oxide without cations in the tetrahedral site. Appl. Phys. Lett. 96, 182103 (2010).

32. Ryden, W., Lawson, A. \& Sartain, C. Electrical transport properties of $\mathrm{IrO}_{2}$ and $\mathrm{RuO}_{2}$. Phys. Rev. B 1, 1494-1500 (1970).

33. Mattheiss, L. Electronic structure of $\mathrm{RuO}_{2}, \mathrm{OsO}_{2}$, and $\mathrm{IrO}_{2}$. Phys. Rev. B 13, 2433-2450 (1976).

34. Hirata, Y. et al. Complex orbital state stabilized by strong spin-orbit coupling in a metallic iridium oxide $\mathrm{IrO}_{2}$. Phys. Rev. B 87, 161111 (2013).

35. Idzuchi, H., Fukuma, Y., Wang, L. \& Otani, Y. Spin relaxation mechanism in silver nanowires covered with $\mathrm{MgO}$ protection layer. Appl. Phys. Lett. 101, 022415 (2012).

36. Fukuma, Y. et al. Giant enhancement of spin accumulation and long-distance spin precession in metallic lateral spin valves. Nat. Mater. 10, 527-531 (2011)

37. Abe, Y., Hasegawa, T., Kawamura, M. \& Sasaki, K. Characterization of Ag oxide thin films prepared by reactive RF sputtering. Vacuum 76, 1-6 (2004).

38. Valenzuela, S. O. \& Tinkham, M. Spin-polarized tunneling in roomtemperature mesoscopic spin valves. Appl. Phys. Lett. 85, 5914 (2004).

39. Idzuchi, H., Fukuma, Y., Wang, L. \& Otani, Y. Spin diffusion characteristics in magnesium nanowires. Appl. Phys. Express 3, 063002 (2010).

40. Althammer, M., Karrer-Müller, E.-M., Goennenwein, S. T. B., Opel, M. \& Gross, R. Spin transport and spin dephasing in zinc oxide. Appl. Phys. Lett. 101, 082404 (2012).

41. Suzuki, A., Matsushita, T., Wada, N., Sakamoto, Y. \& Okuda, M. Transparent conducting Al-doped $\mathrm{ZnO}$ thin films prepared by pulsed laser deposition. Jpn J. Appl. Phys. 35, L56-L59 (1996).

42. Shitade, A. et al. Quantum spin Hall effect in a transition metal oxide $\mathrm{Na}_{2} \mathrm{IrO}_{3}$. Phys. Rev. Lett. 102, 256403 (2009).

43. Wan, X., Turner, A. M., Vishwanath, A. \& Savrasov, S. Y. Topological semimetal and Fermi-arc surface states in the electronic structure of pyrochlore iridates. Phys. Rev. B 83, 205101 (2011).

44. Jackeli, G. \& Khaliullin, G. Mott insulators in the strong spin-orbit coupling limit: from Heisenberg to a quantum compass and Kitaev models. Phys. Rev. Lett. 102, 017205 (2009). 


\section{Acknowledgements}

We thank K. Ohgushi for helpful discussions. This work was supported by Grants-in-Aid (number 22340108, 23103518 and 24224010) from MEXT, Japan.

\section{Author contributions}

K.F., Y.F. and J.M. designed the experiments. K.F., Y.F. and H.I. fabricated devices and collected the data. K.F. and Y.N. analysed the data. K.F. wrote the manuscript with input from J.M. Y.O., and H.T. H.T. and Y.O. planned and supervised the project. All authors discussed the results.

\section{Additional information}

Supplementary Information accompanies this paper at http://www.nature.com/ naturecommunications

Competing financial interests: The authors declare no competing financial interests.

Reprints and permission information is available online at http://npg.nature.com/ reprintsandpermissions/

How to cite this article: Fujiwara, K. et al. $5 d$ iridium oxide as a material for spin-current detection. Nat. Commun. 4:2893 doi: 10.1038/ncomms3893 (2013). 